\title{
Aprendizaje de hábitos posturales y de ejercicio físico saludables en niños sanos con problemas leves de columna vertebral
}

\author{
G. Galindo Morales ${ }^{a}$, MP. Lalana Josa ${ }^{b}$, MB. Sola Martínez ${ }^{c}$, J. Sola Antón ${ }^{d}$ \\ aFisioterapeuta. ${ }^{b P e d i a t r a . ~}{ }^{C T}$ Trabajo Social. CS Borja. Servicio Aragonés de Salud. Sector III. Borja, Zaragoza. España. \\ ¿Técnico en Diagnóstico por la Imagen. CS Canal Imperial. Servicio Aragonés de Salud. Sector II. Zaragoza. España.
}

\section{Resumen}

Objetivos: elaborar y desarrollar un programa que aumente los conocimientos de los niños y sus familias sobre una correcta higiene postural vertebral, establecer un hábito de ejercicio físico e implicar a los niños en su autocuidado para prevenir futuras patologías de espalda.

Material y métodos: se incluyó a 13 niños en los que se detectaron problemas leves de espalda (con o sin dolor) en un programa elaborado conjuntamente por el Servicio de Pediatría y de Fisioterapia. El programa consistía en una valoración inicial con enseñanza individualizada de ejercicios y, posteriormente, tres reuniones grupales: en la primera se entregó una encuesta inicial y un cuaderno de actividades; en las dos posteriores, se valoraron las actividades realizadas, se mostró un vídeo de higiene postural y se pusieron en común los conocimientos adquiridos. A los tres meses se realizó una exploración final y una encuesta de satisfacción a los que completaron el programa.

Resultados: de los 13 participantes terminaron el programa 6 (46,1\%). En la exploración al finalizar el programa, el 100\% había mejorado la flexibilidad vertebral y muscular, y habían aumentado sus conocimientos sobre higiene postural y actividad física. En todos la encuesta de satisfacción fue favorable.

Conclusiones: en los niños que completaron el programa se objetiva mejoría en la exploración. La tasa de abandono es mayor a mayor edad. La mejor edad para aplicar el programa estaría entre los 10-12 años.

Palabras clave: Dolor de espalda. Higiene postural. Fisioterapia. Atención Primaria.

The learning of healthy postural habits and physical exercise in children with slight problems of the vertebral column

Abstract

Objectives: to elaborate and develop a program aimed to increase the child and his/her family's knowledge of a correct postural vertebral hygiene, to establish habits of physical

Gema Galindo Morales: ggalindo@salud.aragon.es

Los autores declaran no presentar conflictos de intereses en relación con la preparación y publicación de este artículo. 
exercise in the child, and to involve him/her in self-care so as to prevent back problems in the future.

Material and methods: thirteen children in whom slight problems of back were detected (with or without pain) were included in a program jointly elaborated by the Pediatrics and the Physical Therapy services. This program consisted of an initial evaluation and individualized teaching of exercises. This was followed by three group meetings: in the first, an initial survey and a notebook of activities were given to the children and families enrolled; in the next two, the activities carried out were evaluated. A video of postural hygiene was shown and the acquired knowledge was jointly discussed. Three months later, a final exploration and satisfaction survey was conducted with those children who had completed the program.

Results: of the 13 initial participants, 6 finished the program (46.1\%). In the exploration carried out to those who had finished the program, 100\% had improved their vertebral and muscular flexibility and had increased their knowledge of postural hygiene and physical activity. In all, the survey of satisfaction was favorable.

Conclusions: the exploration showed an improvement in the children who completed the program. The older the child, the higher the rate of abandonment. The best age for the application of the program could be between 10-12 years.

Key words: Back pain. Postural hygiene. Physical therapy. Primary Care.

\section{Introducción}

Con frecuencia en las revisiones pediátricas del niño sano o consultas a demanda se detectan problemas de espal$\mathrm{da}^{1}$ que, sin ser patológicos, generan dolor, contracturas y la posibilidad en la edad adulta de problemas de mayor importancia.

Muchos de estos problemas son generados por malos hábitos posturales², falta de ejercicio físico ${ }^{3}$ y sobrepeso ${ }^{4}$.

Dado que muchas veces la motivación de la población infantil para el ejercicio físico es escasa y con la finalidad de conseguir que los niños consigan establecer buenos hábitos, se plantea la realización de un programa dirigido al niño y supervisado por su familia, tutelando las actividades a realizar e implicando directamente al propio niño para conseguir logros en la mejora de su salud.

Los objetivos principales planteados son:

1. Aumentar los conocimientos del niño y de su familia sobre la columna vertebral $^{5}$ y su higiene postural en las actividades de la vida diaria ${ }^{6}$.

2. Establecer un hábito diario de ejercicio en el niño a través de la realización de ejercicios de espalda, eliminación de hábitos sedentarios y preferiblemente iniciación en un deporte ${ }^{3}$.

3. Mejorar la actitud postural y/o la estática vertebral del niño a través de técnicas de equilibración muscular con- 
siguiendo con ello disminuir o eliminar el posible dolor.

4. Implicar al niño en el autocuidado de su espalda como medida de prevención ante futuras patologías.

\section{Material y métodos}

\section{Selección de pacientes}

Se lleva a cabo en la consulta de Pediatría durante la revisión del programa

Tabla 1. Detalle de la hoja de exploración fisioterápica inicial

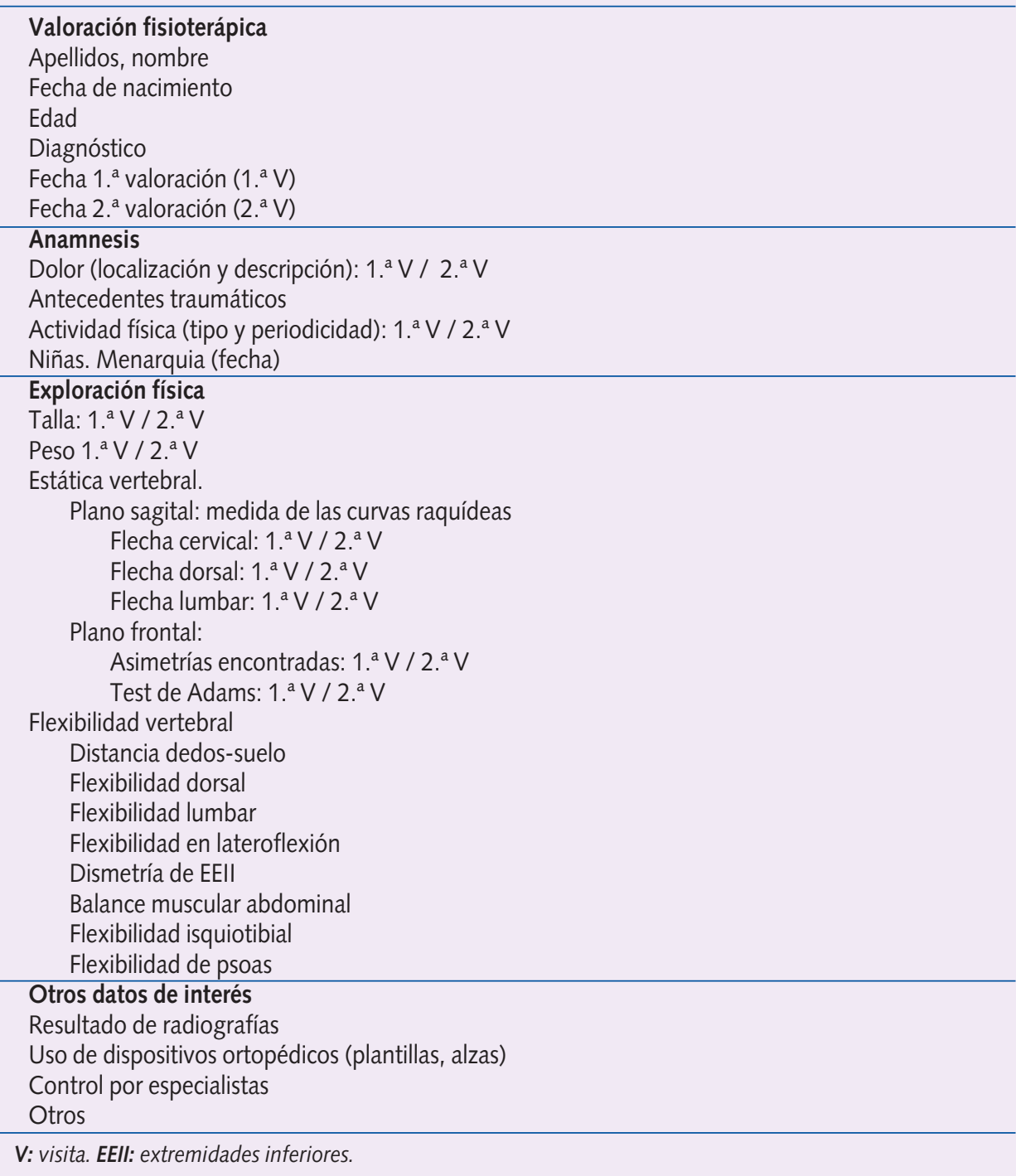


de salud infantil, y en consulta a demanda por dolor de espalda.

Se explican al familiar los objetivos, métodos y duración del programa. Los criterios de inclusión que deben cumplirse son:

1. Niños entre 10-14 años.

2. Presencia o no de dolor vertebral.

3. Alteración de la estática vertebral en el plano frontal (escoliosis) o sagital (hiperlordosis, hipercifosis), ya sea actitud o deformidad estructurada leve ${ }^{7}$.

4. Patología no susceptible de intervención a nivel especializado.
5. Aceptación del programa por el niño y su familia.

Todos los pacientes son derivados a Traumatología para descartar la necesidad de intervención especializada.

Con la aceptación del programa por parte de los usuarios, son derivados a Fisioterapia.

\section{Valoración inicial}

Se realiza en la consulta de Fisioterapia. Consta de cuatro apartados:

1. Encuesta inicial sobre conocimientos de higiene postural vertebral. Cons-

\section{Programa de aprendizaje de hábitos posturales saludables.}

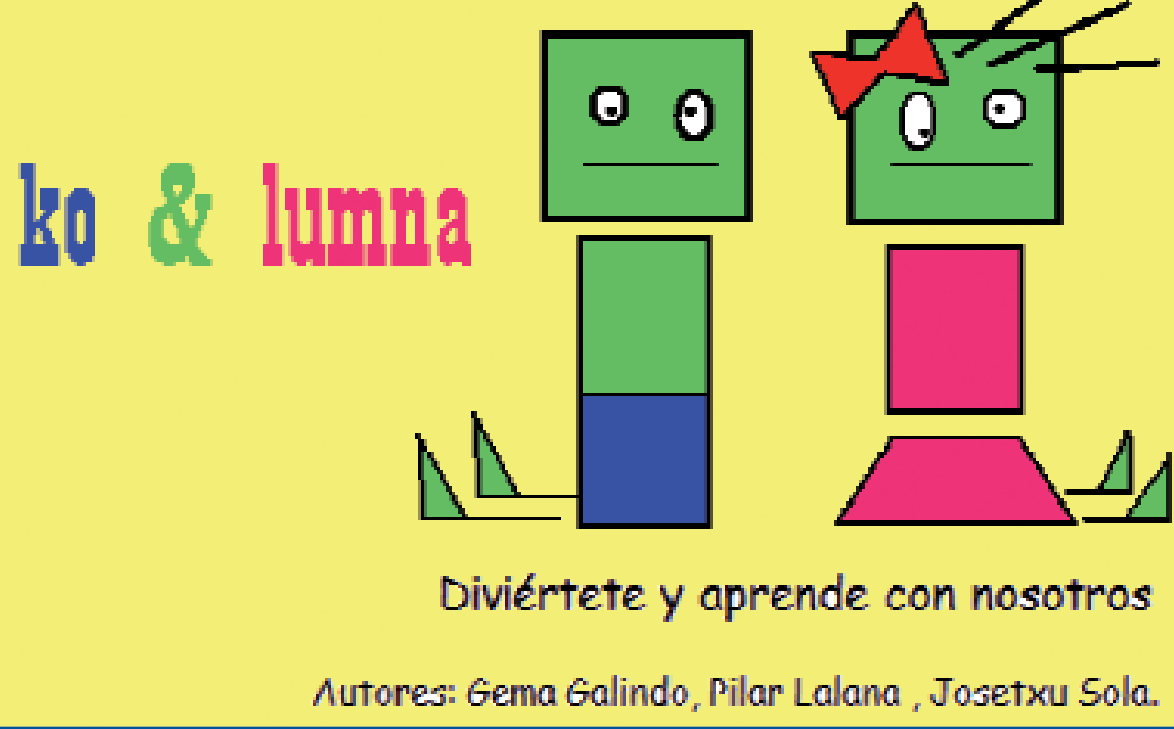


ta de una única pregunta que contestan individualmente el niño y el progenitor: "Enumera hábitos que realizas diariamente que sean beneficiosos para tu espalda".

2. Exploración fisioterápica inicial ${ }^{7-9}$ (tabla 1).

3. Elaboración y enseñanza de un programa de ejercicios adaptado según los hallazgos encontrados en la exploración y por tanto individualizado.

4. Emplazamiento para una reunión grupal con todos los niños que participan en el programa.

\section{Métodos de trabajo complementarios}

Para una mejor comprensión por parte del niño se elabora con anterioridad al inicio del programa un material didáctico adaptado a ellos que consiste en un cuaderno de trabajo y un audiovisual de higiene postural.

\section{Cuaderno de trabajo}

Se elabora en formato papel para que los niños puedan conservar la información y consultarla una vez finalizado el programa.

Tabla 2. Cuaderno de trabajo. Información del primer tema: "Conoce tu columna vertebral"

\section{Conoce tu columna vertebral}

La columna vertebral está situada en la línea media del tronco y está formada por 33-34 vértebras que al unirse entre sí por los discos vertebrales forman las articulaciones intervertebrales.

Las vértebras no son todas iguales. Podemos dividir la columna en cinco regiones: cervical, dorsal, lumbar, sacra y coccígea.

$\square$ Realiza una búsqueda y averigua cuántas vértebras forman cada región y localiza estas regiones en el dibujo que se adjunta.

La columna vertebral no es recta. Posee unas curvaturas que hacen que sea más resistente a las presiones. Es muy importante mantener esas curvaturas en todo momento.

Además de los discos intervertebrales, hay ligamentos que refuerzan la unión entre las vértebras; y músculos, que rodean a la columna y además de ayudar a unir las vértebras, producen el movimiento.

Una sola articulación vertebral apenas tiene movimiento, pero todas ellas en conjunto hacen posible que la columna se mueva.

Es muy importante mantener la musculatura de la columna en condiciones óptimas para repartir los esfuerzos y repercutir lo menos posible en nuestra columna.

$\square$ Pregunta a 5 adultos si tienen o han tenido alguna vez dolor de espalda. ¿Cuántas veces? Anota su nombre y su respuesta. 
Se crean dos personajes, "Ko y Lumna" (figura 1), que de una forma atractiva (cómic) facilitan las explicaciones y les proponen ejercicios. El cuaderno se estructura en seis grandes temas: conoce tu columna vertebral5; ; ejercicio físico y actividad deportiva3 ${ }^{3}$; comer sano tam- bién es importante ${ }^{3}$; transportar la mochila ${ }^{10-12}$; estar sentado $0^{4,13} y$ otras recomendaciones de higiene postural ${ }^{1,6}$. Cada tema consta de tres apartados diferenciados:

1. Información sobre el tema a tratar para ser leída por los niños. En el primer

Figura 2. Cuaderno de trabajo. Actividades a realizar por el niño.
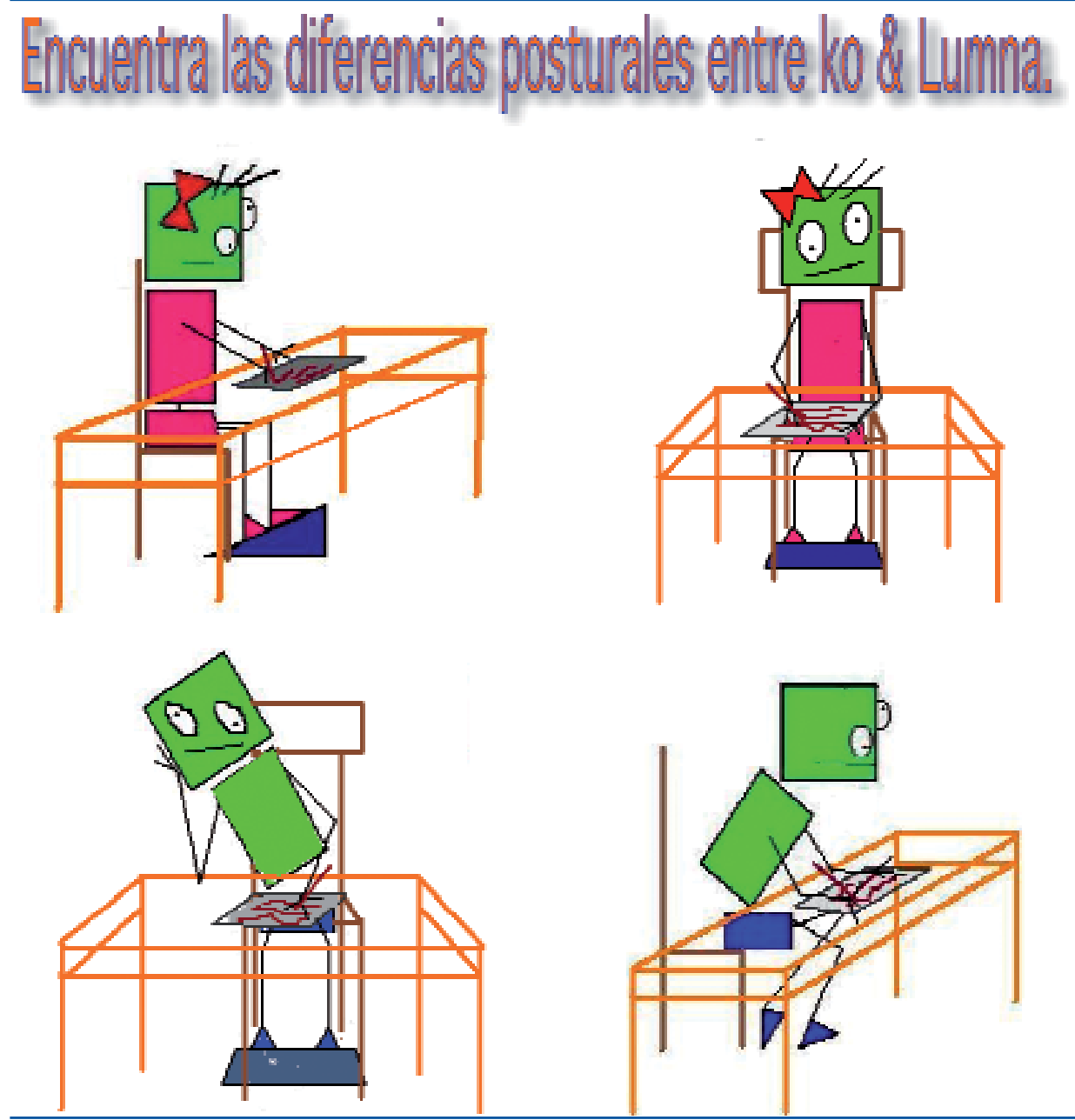
tema ("Conoce tu columna vertebral"), por ejemplo, se habla sobre el número de vértebras, las diferentes regiones vertebrales, las articulaciones, discos, ligamentos y músculos que rodean la columna; el movimiento que se genera y la importancia de mantener la musculatura en condiciones óptimas para repartir esfuerzos y proteger la columna (tabla 2).

2. Actividades relacionadas con el tema. Se les plantean actividades del tipo verdadero o falso, buscar "los siete errores", completar cuadros, contestar preguntas, pesar la mochila, colorear, comparar fotografías del antes y el después de poner en práctica la higiene postural correcta, etc. En el segundo tema "Estar sentado", por ejemplo, se propone encontrar las diferencias posturales entre los personajes del cuaderno de actividades (figura 2).

3. Dos tablas semanales. En ellas el niño anota diariamente la hora de realización de los ejercicios y las dificultades que le han surgido (dolor, fatiga, bienestar, etc.). Dispone, por tanto, de dos semanas para completar cada tema (tabla 3).

\section{Audiovisual de higiene postural}

Se crea en formato digital (DVD) de 15 minutos de duración. Muestra las normas básicas y generales de higiene postural vertebral en postura estática y en las tareas más habituales de la vida diaria, mostrando la forma incorrecta y posteriormente la forma correcta de realización.

Tabla 3. Cuaderno de trabajo. Tabla para anotar la realización de los ejercicios

\section{Semana 1}

Realiza tus ejercicios. Anota la hora y cómo te has sentido (tengo/no tengo dolor, me fatigo, me siento mejor, son fáciles/difíciles...)

Lunes:

Martes:

Miércoles:

Jueves:

Viernes:

\section{Semana 2}

Realiza tus ejercicios. Anota la hora y cómo te has sentido (tengo/no tengo dolor, me fatigo, me siento mejor, son fáciles/difíciles...)

Lunes:

Martes:

Miércoles:

Jueves:

Viernes: 
Incluye información como: tipo de colchón y almohada adecuados; postura correcta al dormir, estar en bipedestación o sedestación ${ }^{4,13}$; colocación y manejo del ordenador ${ }^{14}$ y organización de la mesa de trabajo; levantamiento y transporte de pesos (bolsa, mochila ${ }^{2,10,11}$ o maleta), etc. ${ }^{6}$ (figura 3).

\section{Reuniones grupales}

Se plantean 3 reuniones en grupo con periodicidad mensual, supervisadas por el fisioterapeuta y el pediatra. Las reuniones se llevan a cabo en el gimnasio de Fisioterapia. Los niños pueden acudir solos o acompañados por el familiar. Las tareas desarrolladas en cada reunión grupal son:

Primera reunión: explicación y entrega del cuaderno de trabajo, indicación de completar los dos primeros temas hasta la siguiente reunión y supervisión de la correcta ejecución de los ejercicios.

Segunda reunión: puesta en común y corrección de las actividades del cuaderno de trabajo realizadas, resolución de dudas y preguntas, supervisión de la

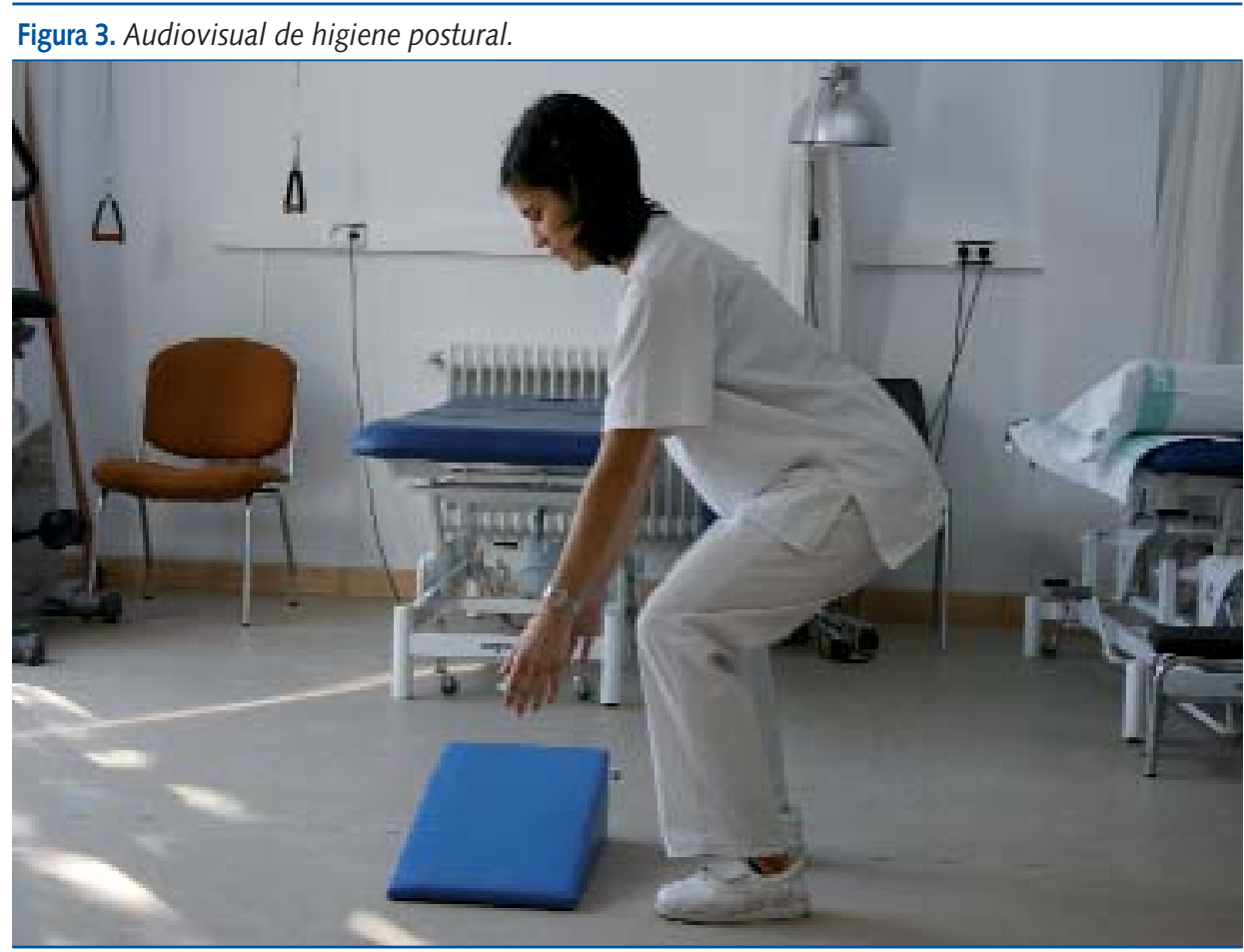


correcta ejecución de los ejercicios y propuesta de realización de los dos temas siguientes.

Tercera reunión: puesta en común y corrección de las actividades del cuaderno de trabajo realizadas, resolución de dudas y preguntas, supervisión de la correcta ejecución de los ejercicios, proyección del audiovisual de higiene postural, propuesta de cumplimentación de los dos últimos temas, estando el último en relación al audiovisual proyectado, entrega de la encuesta final sobre conocimientos de higiene postural adquiridos que consta de una única pregunta que contestan individualmente el niño y el padre/madre ("Enumera hábitos que realizas diariamente que sean beneficiosos para tu espalda") y encuesta de satisfacción del tratamiento para contestar por los padres.

\section{Valoración final}

Se realiza en la consulta de Fisioterapia y consta de 4 apartados:

1. Corrección de los dos últimos temas del cuaderno de trabajo de forma individual.

2. Admisión de la encuesta final sobre conocimientos adquiridos y de la encuesta de satisfacción de tratamiento.

3. Exploración fisioterápica final (mismos ítems que la inicial).
4. Resolución de preguntas del niño y los padres en relación al programa, animándoles en el compromiso de continuar con los autocuidados en el domicilio y prestándonos a la resolución futura de cualquier duda al respecto.

\section{Resultados}

Se propuso realizar el programa a 14 niños de entre 10 y 14 años. Aceptaron participar en él 13 (84,6\% niños, $15,4 \%$ niñas). De ellos, terminaron el programa $6(46,1 \%), 5$ niños $(83,3 \%)$ y 1 niña $(16,7 \%)$.

En la exploración inicial, 2 de ellos presentaban dolor vertebral (33,3\%). El $100 \%$ tenía alteraciones en la estática vertebral en mayor o menor grado (siendo el 83,3\% escoliosis) y desviaciones de los valores normales para la flexibilidad vertebral y muscular.

En la encuesta inicial sobre higiene postural, "Enumera hábitos que realizas diariamente que sean beneficiosos para tu espalda", el 33,3\% no enumeró ningún hábito, el $50 \%$ enumeró uno y el $16,7 \%$ enumeró dos.

En la exploración final, sólo 1 seguía presentando dolor que describió como "ocasional" (16,6\%).

Se mantuvo invariable el porcentaje $(83,3 \%)$ de niños con desviaciones en el plano frontal, pero se mejoraron los 
valores de las medidas para las desviaciones en el plano sagital (cifosis, lordosis) en el $100 \%$ de los pacientes. El $100 \%$ había mejorado los valores de la flexibilidad vertebral y muscular. En la encuesta final sobre higiene postural, un $16,6 \%$ enumeró un hábito saludable y el $83,4 \%$ enumeró dos o más.

La encuesta de satisfacción para padres fue positiva en el $100 \%$ de los casos.

\section{Discusión}

El $100 \%$ de los niños que no terminaron el programa eran mayores de 12 años. Observamos que los niños más mayores eran más reticentes a acudir a las sesiones, iban normalmente solos, eran menos receptivos y participaban menos en las actividades. Todo ello nos lleva a pensar que la edad adecuada para desarrollar el programa estaría entre los 10 y los 12 años. A esta edad normalmente todavía les acompaña un progenitor, el niño y la familia se implican más, son más dóciles y cuanto antes se establezcan los buenos hábitos y la realización de un deporte, mayores probabilidades habrá de conseguir y mantener cambios a largo plazo.

Pensamos que reduciendo los grupos a 6-8 niños se aseguraría un seguimiento más personalizado y se reduci- ría la tasa de abandono. Con menos niños y de edades más afines, probablemente la participación sea mayor y se consideren las actividades más como algo lúdico que como una obligación; se establecen mejor los vínculos entre los profesionales, las familias y los niños, y la sesión termina siendo más amena y entretenida y con grado máximo de implicación.

En el $100 \%$ de los casos hubo una mejoría objetiva en la exploración. Al reevaluar a los niños y pese a que consideramos que el tiempo que duró el programa es corto, la mejoría fue evidente en todos los niños. Todos ellos manifestaron encontrarse mejor, consideraban que el cumplimiento del programa no les había resultado costoso y hasta les había divertido y pensaban seguir intentando aplicar lo aprendido.

Todos los que terminaron el programa habían aumentado sus conocimientos sobre higiene postural. La encuesta de satisfacción fue favorable en todos los casos y en el apartado de sugerencias dos de ellos planteaban que estaría bien hacer un seguimiento durante más tiempo.

Estos datos responden a los objetivos principales planteados al inicio del programa, obteniendo la consecución de todos ellos salvo el de iniciación en un 
deporte, que se establece como un objetivo a largo plazo supeditado al inicio del curso escolar, puesto que el programa se desarrolló durante las vacaciones de verano para facilitar la asistencia y el cumplimiento por parte de los niños.

Dado que el programa no contempla un seguimiento del niño para valorar si continúa poniendo en práctica los hábitos aprendidos (realización de ejercicios e higiene postural), no es posible precisar cuánto tiempo se mantendrá la mejora obtenida en la exploración. Este dato nos hace pensar en la inclusión de un apartado de seguimiento en futuros desarrollos del programa.

\section{Agradecimientos}

A Susana Ónega por su ayuda en la preparación del manuscrito.

\section{Bibliografía}

1. Blanquer Ropero JJ, Rodríguez-Piñero Durán $M$, Ibáñez Campos T, Expósito Tirado JA. Dolor de espalda en el niño y el adolescente. Rev Pediatr Integral. 2002;6:503-14.

2. Castillo Sánchez MD, Naranjo Rodríguez $J A$, León Espinosa MT. Experiencia educativa sobre hábitos posturales saludables. Rev SEMG. 2003;55:400-6.

3. Casado Pereza C, Alonso Fernández N, Hernández Barrera V, Jiménez García R. Actividad física en niños españoles. Factores asociados y evolución 2003-2006. Rev Pediatr Aten Primaria. 2009;11:219-31.

4. Fraile García PA. Dolor de espalda en alumnos de primaria y sus causas. Fisioterapia. 2009;31:137-42.

5. Miralles Marrero RC. Biomecánica clínica del aparato locomotor. Barcelona: Masson; 1998.

6. La Web de la espalda. Fundación Kovacks. [Internet]. Disponible en www.webdelaespal da.org/divulgativa/su_espalda/escolares.asp [consultado el 20/01/2010].

7. Elías Pollina J. Exploración del raquis: valo- ración de las alteraciones y enfoque diagnóstico. Rev Pediatr Integral. 2002;6:491-8.

8. Backup K. Pruebas clínicas para patología ósea, articular y muscular. Barcelona: Masson, 1997.

9. King HA. Back pain in children. En: Weinstein SL. The pediatric spine: principles and practice. New York: Raven Press Ltd.; 1994. p. 173-83.

10. Ruano Aguilar JM, Palafox Sánchez R, García Buenrostro N. Las mochilas en los escolares y su asociación con dolor de espalda. Acta Médica Grupo Ángeles. 2007;5:225-7.

11. Bort Saborit N, Simó Pitarch A. Carritos o mochilas en la edad escolar. Fisioterapia. 2002; 24:63-72.

12. Brewer JM, Davis KG, Dunning KK, Succop PA. Does ergonomic mismatch at school impact pain in school children? Work. 2009;34:455-64.

13. Quintana E, Marín A, Sánchez C, Rubio I, López N, Calvo Jl. Estudio de la postura sedente en una población infantil. Fisioterapia. 2004;26:153-63.

14. Gismera Neuberger S. Ordenadores y niños. Madrid: Pirámide, 1996. 